\title{
MicroRNA-466 inhibits cell proliferation and invasion in osteosarcoma by directly targeting insulin receptor substrate 1
}

\author{
YONGNING SUN ${ }^{1 *}$, JINGZHEN ZHOU $^{2 *}$, LINA SHI $^{1},{\text { JIE } \mathrm{LI}^{2} \text { and JIANMING CHEN }}^{3}$ \\ ${ }^{1}$ Department of Clinical Laboratory, Ningbo No. 6 Hospital; ${ }^{2}$ Department of Emergency, \\ Ningbo No. 2 Hospital; ${ }^{3}$ Department of Orthopedics, Ningbo No. 6 Hospital, Ningbo, Zhejiang 315040, P.R. China
}

Received August 2, 2018; Accepted January 29, 2019

DOI: $10.3892 / \mathrm{mmr} .2019 .9956$

\begin{abstract}
Accumulating evidence has demonstrated that microRNAs (miRNAs) are frequently dysregulated in osteosarcoma (OS), and the aberrant expression of miRNAs is associated with OS initiation and progression. Previous studies demonstrated that miRNA-466 (miR-466) is dysregulated, and serves important roles in various types of human cancer. However, the role of miR-466 in the formation and progression of OS remains unclear. In the present study, the expression level of miR-466 was identified to be markedly downregulated in OS tissues and cell lines. Additionally, miR-466 overexpression inhibited the proliferative and invasive abilities of OS cells. In the present study, bioinformatics analyses and luciferase assays were employed to show that miR-466 was able to directly target the 3'-untranslated region of insulin receptor substrate 1 (IRS1) gene, negatively regulating the mRNA and the protein expression levels of IRS1 in OS cells. Furthermore, IRS1 was upregulated in OS tissues, and the increased expression level of IRS1 exhibited an inverse correlation with the expression level of miR-466. Furthermore, IRS1 overexpression was able to partially reverse the suppressive effects of miR-466 overexpression in OS cells. To the best of the authors' knowledge, the present study is the first to suggest that miR-466 is downregulated in OS and inhibits the progression of OS by directly targeting IRS1. The present results suggested that miR-466 may represent a novel potential therapeutic target for the treatment of patients with OS.
\end{abstract}

\section{Introduction}

Osteosarcoma (OS) derives from mesenchymal cells and is the most common primary malignant bone cancer (1). The

Correspondence to: Professor Jianming Chen, Department of Orthopedics, Ningbo No. 6 Hospital, 1059 Zhongshan Road, Ningbo, Zhejiang 315040, P.R. China

E-mail: jmchen_orth@126.com

${ }^{*}$ Contributed equally

Key words: microRNA-466, osteosarcoma, proliferation, invasion, insulin receptor substrate 1 incidence rate of OS is $\sim 4.4$ per million worldwide, and incidence is higher in adolescents (15-19 years old) (2). OS accounts for $\sim 5 \%$ of all malignant tumors in children and adolescents (3). The principal therapeutic strategies for patients with OS are surgical resection, radiotherapy and multidrug chemotherapy (4). Although substantial improvements have been achieved in the treatment of OS, the therapeutic outcomes remain poor due to the high recurrence rate and the metastatic features of OS (5). Various factors, including genetic mutations and environmental ionizing radiation, are associated with the development and progression of OS (6). However, the molecular mechanisms underlying OS pathogenesis remain unclear. Therefore, improving the understanding of the molecular processes underlying the initiation and development of OS may contribute to the identification of novel therapeutic strategies for the treatment of patients with this malignancy.

microRNAs (miRNAs) are short (18-25 nucleotides in length) noncoding RNA molecules that are able to directly bind to the 3'-untranslated regions (3'-UTRs) of their target genes inducing translational inhibition and/or mRNA degradation (7). In total, $>1,000$ miRNAs have been identified in the human genome (8). miRNAs account for $3 \%$ of all human genes; however, these short noncoding RNAs are able to modulate the expression levels of $\sim 66 \%$ of all coding human genes (8). Accumulating evidence has demonstrated that the dysregulation of miRNAs is frequent in human malignancies, and the altered expression level of miRNAs was identified to be important in maintaining the malignant phenotype of certain tumor cells (9-11). In particular, various miRNAs were identified to be downregulated in OS, including miR-103 (12), miR-187 (13) and miR-365 (14). These downregulated miRNAs may serve as tumor suppressors or oncogenes based on the biological function of their target genes (15). Therefore, the identification of novel miRNAs involved in OS initiation and development may contribute to the development of effective therapeutic strategies to improve the outcomes of patients with OS.

Previous studies demonstrated that miRNA-466 (miR-466) is dysregulated in various types of human cancer, and it was identified to be associated with cancer progression (16-18). However, whether miR-466 is involved in the initiation and progression of OS remains unclear. Therefore, the present study aimed to examine the expression level of miR-466 in OS tissues and cell lines, investigating the function and the 
molecular mechanisms associated with miR-466 in OS in order to identify a novel potential therapeutic target to treat patients with OS.

\section{Materials and methods}

Tissue collection. The present study was approved by The Ethics Committee of The Ningbo No. 6 Hospital. Written informed consent was obtained from all patients for the use of their clinical tissues. In total, 26 pairs of OS and matched adjacent normal tissues were collected from patients (17 males, 9 females; age range, 13-45 years) who received surgical resection at The Ningbo No. 6 Hospital between March 2015 and June 2017. Patients who received radiotherapy or chemotherapy treatment before surgery were excluded from the study. The collected tissues were snap-frozen in liquid nitrogen and stored at $-80^{\circ} \mathrm{C}$ until further analysis.

Cell lines. A normal human osteoblast (hFOB1.19) and four human OS (SAOS-2, HOS, MG-63 and U2OS) cell lines were purchased from The American Type Culture Collection (Manassas, VA, USA). Dulbecco's modified Eagle's medium (DMEM) supplemented with 10\% fetal bovine serum (FBS), $100 \mathrm{U} / \mathrm{ml}$ penicillin and $100 \mu \mathrm{g} / \mathrm{ml}$ streptomycin (all from Gibco; Thermo Fisher Scientific, Inc., Waltham, MA, USA) was used to culture the cell lines. All cells were maintained at $37^{\circ} \mathrm{C}$ in a $5 \% \mathrm{CO}_{2}$ humidified atmosphere.

Cell transfection. The miR-466 mimics and miRNA negative control mimics (miR-NC) were purchased from Shanghai GenePharma Co., Ltd. (Shanghai, China). To promote miR-466 overexpression, cells were seeded into 6-well plates with a density of $6 \times 10^{5}$ cells/well one day prior to transfection. Subsequently, miR-466 mimics and miR-NC were transfected. IRS1 overexpression plasmid pCMV-IRS1 and the empty control plasmid pCMV were purchased from Hunan Nanhua Ai Shi Purin Biotechnology Co., Ltd. (Changsha, China). pCMV-IRS1 was transfected to overexpress IRS1, whereas empty pCMV plasmid was used as the negative control. Cells were transfected with miR-466 mimics (100 pmol), miR-NC $(100 \mathrm{pmol}), \mathrm{pCMV}-\mathrm{IRS} 1(4 \mu \mathrm{g})$ or pCMV $(4 \mu \mathrm{g})$ using Lipofectamine 2000 (Invitrogen; Thermo Fisher Scientific, Inc.) according to the manufacturer's protocol. Following an 8 -h incubation at $37^{\circ} \mathrm{C}$, the culture medium was replaced with fresh DMEM containing 10\% FBS. Cell Counting kit-8 (CCK-8) assay, reverse transcription-quantitative polymerase chain reaction (RT-qPCR) and cell invasion assay were conducted after 24,48 and $48 \mathrm{~h}$, respectively. A total of $72 \mathrm{~h}$ post-transfection, western blot analysis was performed.

$R N A$ isolation and RT-qPCR. Total RNA was isolated from cells and clinical tissues using TRIzol ${ }^{\circledR}$ reagent (Invitrogen; Thermo Fisher Scientific, Inc.) according to the manufacturer's protocol. For miR-466 detection, total RNA was reverse-transcribed using a TaqMan microRNA RT kit (Applied Biosystems; Thermo Fisher Scientific, Inc.). The temperature protocol for RT was as follows: $16^{\circ} \mathrm{C}$ for $30 \mathrm{~min}$, $42^{\circ} \mathrm{C}$ for $30 \mathrm{~min}$ and $85^{\circ} \mathrm{C}$ for $5 \mathrm{~min}$. The expression level of miR-466 was measured using a TaqMan microRNA PCR kit (Applied Biosystems; Thermo Fisher Scientific, Inc.), and
RNA, U6 small nuclear 6 was used as the internal control to normalize the expression level of miR-466. The cycling conditions were as follows: $50^{\circ} \mathrm{C}$ for $2 \mathrm{~min}, 95^{\circ} \mathrm{C}$ for $10 \mathrm{~min}$; followed by 40 cycles of denaturation at $95 \mathrm{C}$ for $15 \mathrm{sec}$ and annealing/extension at $60^{\circ} \mathrm{C}$ for $60 \mathrm{sec}$; and one cycle of final extension at $60^{\circ} \mathrm{C}$ for $60 \mathrm{sec}$ and $4^{\circ} \mathrm{C}$ for $30 \mathrm{sec}$. For the quantification of the mRNA expression level of IRS1, cDNA was synthesized from total RNA using a M-MLV reverse transcriptase (Takara Biotechnology Co., Ltd., Dalian, China), and the cDNA was subsequently amplified using a SYBR Premix Ex Taq II kit (Takara Biotechnology Co., Ltd., Dalian, China). GAPDH was used as the internal reference gene to normalize the expression level of IRS1. The RT reaction comprised $100 \mathrm{ng}$ total RNA, $2.5 \mu$ l primers $(2.5 \mu \mathrm{M}), 7.5 \mu 1$ RNase-free $\mathrm{H}_{2} \mathrm{O}, 4 \mu \mathrm{l}$ 5X RT Buffer, $2 \mu \mathrm{l}$ dNTP Mix, $2 \mu \mathrm{l} 100 \mathrm{mM}$ DTT and $0.5 \mu \mathrm{l}$ MMLV Reverse Transcriptase. The temperature protocol for RT was as follows: $95^{\circ} \mathrm{C}$ for $2 \mathrm{~min}$; followed by 20 cycles at $94^{\circ} \mathrm{C}$ for $1 \mathrm{~min}, 55^{\circ} \mathrm{C}$ for $1 \mathrm{~min}$ and $72^{\circ} \mathrm{C}$ for $2 \mathrm{~min}$; and $72^{\circ} \mathrm{C}$ for $5 \mathrm{~min}$. The PCR cycling conditions were as follows: $5 \mathrm{~min}$ at $95^{\circ} \mathrm{C}$, followed by 40 cycles at $95^{\circ} \mathrm{C}$ for $30 \mathrm{sec}$ and $65^{\circ} \mathrm{C}$ for $45 \mathrm{sec}$; and one cycle of final extension at $95^{\circ} \mathrm{C}$ for $30 \mathrm{sec}, 65^{\circ} \mathrm{C}$ for $45 \mathrm{sec}$ and $4^{\circ} \mathrm{C}$ for $30 \mathrm{sec}$. Data analysis was performed using the $2^{-\Delta \Delta C q}$ quantification method (19). The primers were designed as follows: miR-466, forward 5'-ATGGTTCGTGGG ATACACATACACGCA-3', reverse 5'-GCAGGGTCCGAG GTATTC-3'; U6, forward 5'-GCTTCGGCAGCACATATA CTAAAAT-3', reverse 5'-CGCTTCACGAATTTGCGTGTC AT-3'; IRS1, forward 5'-CAGGCAGAATGAAAGACCTAA ATG-3', reverse 5'-AGACGTGAGGTCCTGGTTGTG-3'; and GAPDH, forward 5'-CAAGGTCATCCATGACAACTTTG-3' and reverse 5'-GTCCACCACCCTGTTGCTGTAG-3'.

CCK-8 assay. The proliferative ability of OS cells was determined using the CCK- 8 assay. The transfected cells were collected at $48 \mathrm{~h}$ post-transfection and plated into 96-well plates at a density of $3 \times 10^{3}$ cells/well. Following incubation for 0, 24, 48 and 72 h, $10 \mu$ l CCK-8 reagent (Dojindo Molecular Technologies, Inc., Kumamoto, Japan) was added to each well, and incubated for $2 \mathrm{~h}$ at $37^{\circ} \mathrm{C}$ with $5 \% \mathrm{CO}_{2}$. The absorbance was detected at a wavelength of $450 \mathrm{~nm}$ with an ELISA plate reader (Model 550; Bio-Rad Laboratories, Inc., Hercules, CA, USA).

Cell invasion assay. Transfected cells were harvested following a 48 -h incubation at $37^{\circ} \mathrm{C}$ and resuspended in FBS-free DMEM. In total, $5 \times 10^{4}$ transfected cells were seeded in the upper compartment of Transwell ${ }^{\circledR}$ chambers coated with Matrigel ${ }^{\circledR}$ (all from Becton, Dickinson and Company, Franklin Lakes, NJ, USA). In total, $600 \mu 1$ DMEM containing 10\% FBS was plated in the lower compartments. Following a 24-h incubation, the non-invasive cells were removed from the upper surface of the Transwell chambers using a cotton swab. The invaded cells were subsequently fixed with $100 \%$ methanol at room temperature for $30 \mathrm{~min}$ and stained with $0.5 \%$ crystal violet solution at room temperature for $30 \mathrm{~min}$. The number of invasive cells was counted in five independent fields per well using an inverted light microscope (Olympus Corporation, Tokyo, Japan).

Target prediction of miR-466. miRDB (http://mirdb.org/) and TargetScan (Release 7.2; http://www.targetscan.org/) were used to predict the potential targets of miR-466. 
Luciferase reporter assay. IRS1 was predicted as one of the principal targets of miR-466. To test this interaction, the 3'-UTR of IRS1 containing the wild-type or mutant miR-466 binding sites were synthesized by Shanghai GenePharma Co., Ltd., and inserted into pMIR-GLOTM Luciferase vectors (Promega Corporation, Madison, WI, USA). Cells were plated in 24-well plates and incubated until they reached a confluence of $60-70 \%$. The co-transfection of luciferase reporter plasmids and miR-466 mimics or miR-NC was performed using Lipofectamine ${ }^{\circledR} 2000$ (Invitrogen; Thermo Fisher Scientific, Inc.) according to the manufacturer's protocol. The transfected cells were incubated at $37^{\circ} \mathrm{C}$ under $5 \% \mathrm{CO}_{2}$ for $48 \mathrm{~h}$. Firefly and Renilla luciferase activities were measured using a Dual-Luciferase ${ }^{\circledR}$ Reporter Assay system (cat. no. E1910; Promega Corporation) according to the manufacturer's protocol. Firefly luciferase activity was normalized to Renilla luciferase activity.

Western blot analysis. Total protein was isolated from tissue specimens or cells using radioimmunoprecipitation assay buffer (Beyotime Institute of Biotechnology, Haimen, China). A bicinchoninic protein assay kit (Beyotime Institute of Biotechnology) was used to measure the protein concentration. An equal amount of total protein $(30 \mu \mathrm{g})$ was loaded in each lane, and proteins were separated by $10 \%$ SDS-PAGE gel and transferred to polyvinylidene difluoride membranes (EMD Millipore, Billerica, MA, USA), and subsequently were blocked with $5 \%$ non-fat milk diluted in Tris-buffer containing $0.1 \%$ Tween-20 (TBST) for $2 \mathrm{~h}$ at room temperature. Following blocking, the membranes were incubated overnight at $4^{\circ} \mathrm{C}$ with anti-IRS1 antibody (cat. no. ab40777; 1:1,000; Abcam, Cambridge, UK) or anti-GAPDH antibody (cat. no. ab181603; 1:1,000; Abcam). Following washing with TBST, the membranes were incubated for $2 \mathrm{~h}$ at room temperature with a horseradish peroxidase-conjugated goat anti-rabbit immunoglobulin G secondary antibody (cat. no. ab6721; 1:5,000; Abcam, Cambridge, UK). Following three washes with TBST, the protein signal was visualised using enhanced chemiluminescence-plus reagents (GE Healthcare, Chicago, IL, USA), according to the manufacturer's protocol. The protein expression levels were quantified using Quantity One software (version 4.62; Bio-Rad Laboratories, Inc.).

Statistical analysis. All assays were repeated at least three times, and data are presented as the mean \pm standard deviation. All statistical analyses were performed using SPSS software (version 16; SPSS, Inc., Chicago,IL, USA). Differences between two groups were determined using Student's t-test, whereas one-way ANOVA followed by Student-Newman-Keuls post hoc test was used for the comparison of multiple groups. Spearman's correlation analysis was used to examine the correlation between the expression levels of miR-466 and IRS1 mRNA in OS tissues. $\mathrm{P}<0.05$ was considered to indicate a statistically significant difference.

\section{Results}

Expression level of miR-466 is decreased in OS tissues and cell lines. RT-qPCR was performed to quantify the expression level of miR-466 in the tissue specimens of 26 patients with OS, and matched adjacent normal tissues were used as a control. The results suggested that the expression level of miR-466 was significantly decreased in OS tissues compared with adjacent normal tissues (Fig. 1A). Subsequently, the expression level of miR-466 was detected in four OS cell lines (SAOS-2, HOS, MG-63 and U2OS) and a normal human osteoblast hFOB1.19 cell line. The expression level of miR-466 was significantly decreased in all four OS cell lines compared with hFOB1.19 cells (Fig. 1B). The present results suggested that miR-466 may serve as an oncosuppressor gene in the initiation and development of OS.

miR-466 suppresses the proliferation and invasion of MG-63 and U2OS cells. To investigate the role of miR-466 in OS progression, miR-466 mimics or miR-NC were transfected into OS cells, and the effects of miR-466 overexpression were determined by examining the proliferation rate and invasive ability of OS cells. MG-63 and U2OS cells exhibited a decreased expression level of miR-466 compared with SAOS-2 and HOS cells. Therefore, these two cell lines were used in subsequent experiments. Following transfection, RT-qPCR analysis identified that the expression level of miR-466 was significantly upregulated in MG-63 and U2OS cells following transfection of miR-466 mimics (Fig. 2A). Additionally, CCK- 8 assays suggested that miR-466 overexpression was able to inhibit the proliferation of MG-63 and U2OS cells at 48 and $72 \mathrm{~h}$ (Fig. 2B). Furthermore, cell invasion assays suggested that overexpression of miR-466 was sufficient to suppress the invasive abilities of MG-63 and U2OS cells (Fig. 2C). Collectively, the present data suggested that miR-466 may serve a tumor-suppressive role by inhibiting cell proliferation and invasion of OS cells.

miR-466 inhibits IRS1 expression by directly targeting its 3'-UTR. To further investigate the molecular mechanism underlying the tumor-suppressive function of miR-466 in OS pathology, bioinformatics analysis was performed to identify the potential targets of miR-466. Numerous genes were predicted as potential targets of miR-466, including IRS1, phosphatase and tensin homolog, insulin-like growth factor (IGF)-like family member 1, SRY-box 6 and metadherin. Among these candidates, IRS1 exhibited a miR-466 binding sequence at position $482-489$ of its 3'-UTR (Fig. 3A). IRS1 was selected for further analyses due to the previously observed function of this gene in OS development and progression (20). In order to test the bioinformatics analysis in vitro, a luciferase reporter assay was conducted. Overexpression of miR-466 significantly decreased the reporter activity of pMIR-GLOTM plasmid containing wild-type IRS1 3'-UTR in MG-63 and U2OS cells (Fig. 3B); however, the reporter activity of the plasmid containing mutant IRS1 3'-UTR was unaffected following miR-466 overexpression, suggesting that miR-466 was able to directly target the 3'-UTR of IRS1. Subsequently, the effect of miR-466 overexpression in OS cells on the endogenous expression level of IRS1 was investigated. RT-qPCR and western blot analyses results suggested that miR-466 overexpression decreased the mRNA (Fig. 3C) and the protein (Fig. 3D) expression levels of IRS1 in MG-63 and U2OS cells. Collectively, the present results suggested that IRS1 may be a direct target of miR-466 in OS cells. 

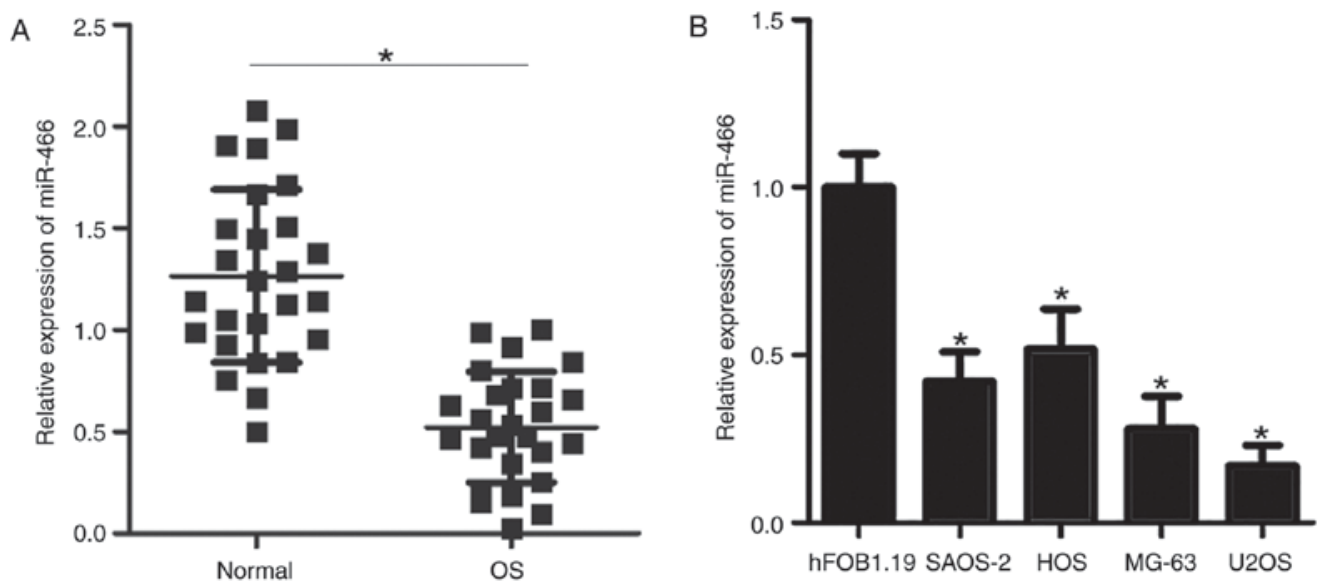

Figure 1. Expression of miR-466 is decreased in OS tissue specimens and cell lines. (A) miR-466 expression was detected by RT-qPCR in 26 pairs of OS and matched adjacent normal tissues. " $\mathrm{P}<0.05$ vs. adjacent normal tissues. (B) Expression level of miR-466 was determined in four human OS cell lines (SAOS-2, HOS, MG-63 and U2OS) and a normal human osteoblast cell line (hFOB1.19) using RT-qPCR. "P<0.05 vs. hFOB1.19. miR-, microRNA; RT-qPCR, reverse transcription-quantitative polymerase chain reaction; OS, osteosarcoma.
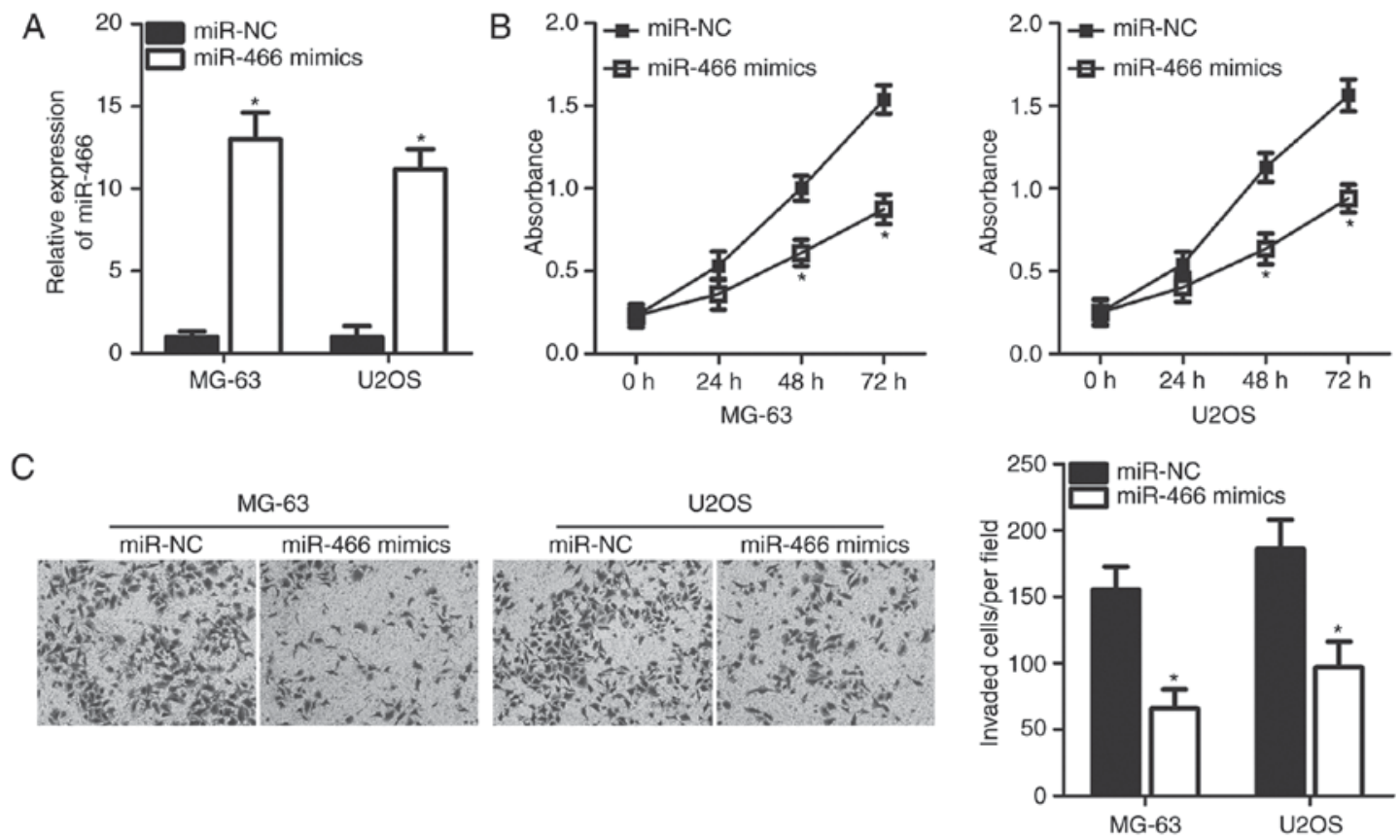

Figure 2. miR-466 overexpression inhibits proliferation and invasion of MG-63 and U2OS cells. (A) miR-466 mimics or miR-NC was transfected into MG-63 and U2OS cells. Reverse transcription-quantitative polymerase chain reaction analysis was performed to detect the transfection efficiency. (B) Cell Counting Kit- 8 assay was used to analyze the effects of miR-466 overexpression on the proliferation of MG-63 and U2OS cells. (C) Matrigel assay was used to measure the invasive abilities of MG-63 and U2OS cells following transfection with miR-466 mimics or miR-NC. Magnification, x200. "P<0.05 vs. miR-NC. miR-, microRNA; NC, negative control.

miR-466 is negatively correlated with IRS1 expression in OS tissues. The aforementioned results suggested that IRS1 may be a direct target gene of miR-466 in OS; therefore, the association between the expression levels of miR-466 and IRS1 was investigated in OS clinical tissues. RT-qPCR analysis suggested that the mRNA expression level of IRS1 was upregulated in OS tissues compared with adjacent normal tissues (Fig. 4A). Subsequently, the protein expression level of IRS1 was detected in various OS tissues and matched adjacent normal tissues using western blot analysis. The protein expression level of IRS1 was increased in OS tissues compared with adjacent normal tissues (Fig. 4B and C). Furthermore,
Spearman's correlation analysis identified an inverse correlation between the mRNA expression levels of miR-466 and IRS1 mRNA in OS tissues (Fig. 4D). The present results suggested that the downregulation of miR-466 may, at least in part, lead to an increase in the expression level of IRS1 in OS tissues.

IRS1 mediates the tumor-suppressing roles of miR-466 in OS cells. Rescue experiments were performed to investigate whether the suppressive effects of miR-466 overexpression in OS cell proliferation and invasion were mediated by IRS1 inhibition. Empty pCMV plasmid or IRS1 overexpression 


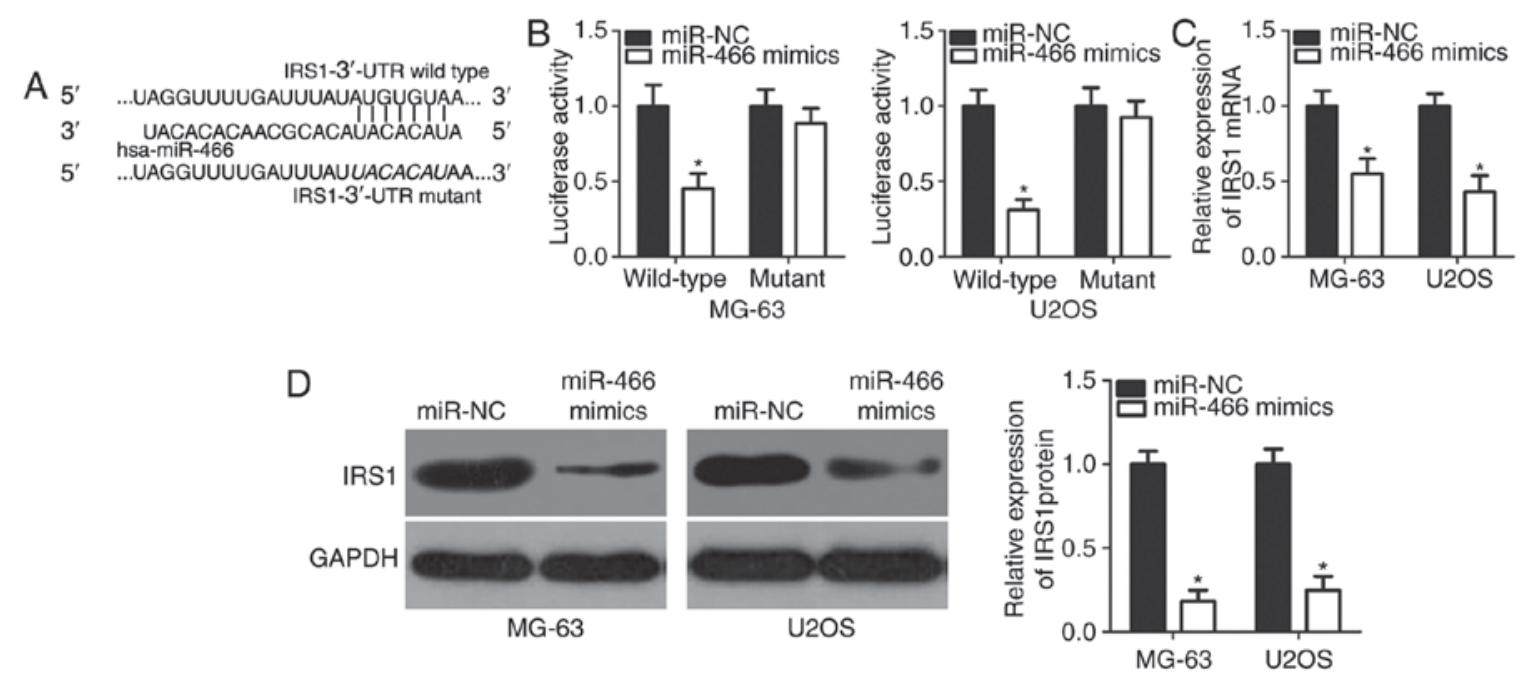

Figure 3. IRS1 is a direct target gene of miR-466 in OS cells. (A) Putative miR-466 wild-type or mutant binding sequences in the 3'-UTR of IRS1. (B) Luciferase activity was detected in MG-63 and U2OS cells that were co-transfected with miR-466 mimics or miR-NC and luciferase reporter plasmid carrying the wild-type or mutant 3'-UTR of IRS1. (C) mRNA and (D) protein expression levels of IRS1 in MG-63 and U2OS cells transfected with miR-466 mimics or miR-NC were examined by reverse transcription-quantitative polymerase chain reaction and western blot analysis, respectively. "P<0.05 vs. miR-NC. miR-, microRNA; IRS1, insulin receptor substrate 1; NC, negative control; UTR, untranslated region.
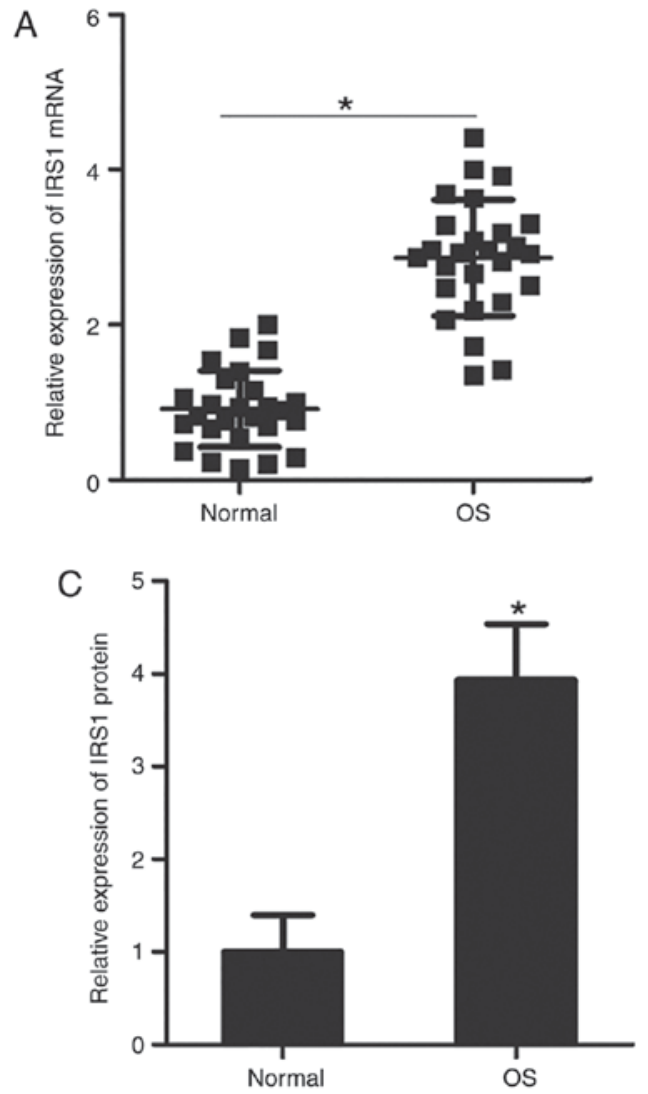
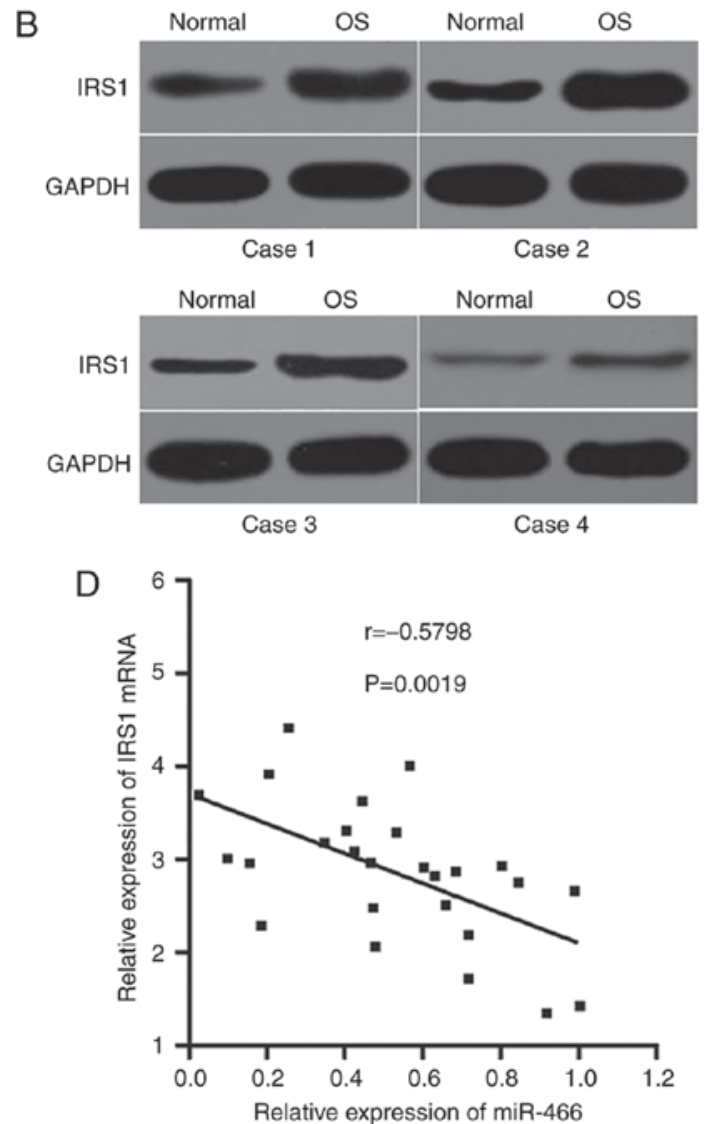

Figure 4. miR-466 expression is inversely correlated with IRS1 in OS tissues. (A) Expression level of IRS1 was upregulated in OS tissues compared with adjacent normal tissues, as determined by reverse transcription-quantitative polymerase chain reaction. Protein expression level of IRS1 in OS tissues, as determined by (B) western blot and (C) densitometry analysis. (D) Spearman's correlation analysis was conducted to evaluate the association between the mRNA expression levels of miR-466 and IRS1 in OS tissues ( $\mathrm{r}=-0.5789 ; \mathrm{P}=0.0019)$. " $\mathrm{P}<0.05$ vs. adjacent normal tissues. OS, osteosarcoma; miR-, microRNA; IRS1, insulin receptor substrate 1 .

plasmid (pCMV-IRS1) without its 3'-UTR was co-transfected with miR-466 mimics into MG-63 and U2OS cells. Western blot analysis suggested that the downregulation of the protein expression level of IRS1 caused by miR-466 overexpression was restored in MG-63 and U2OS cells following co-transfection with pCMV-IRS1 (Fig. 5A). CCK-8 and cell invasion 


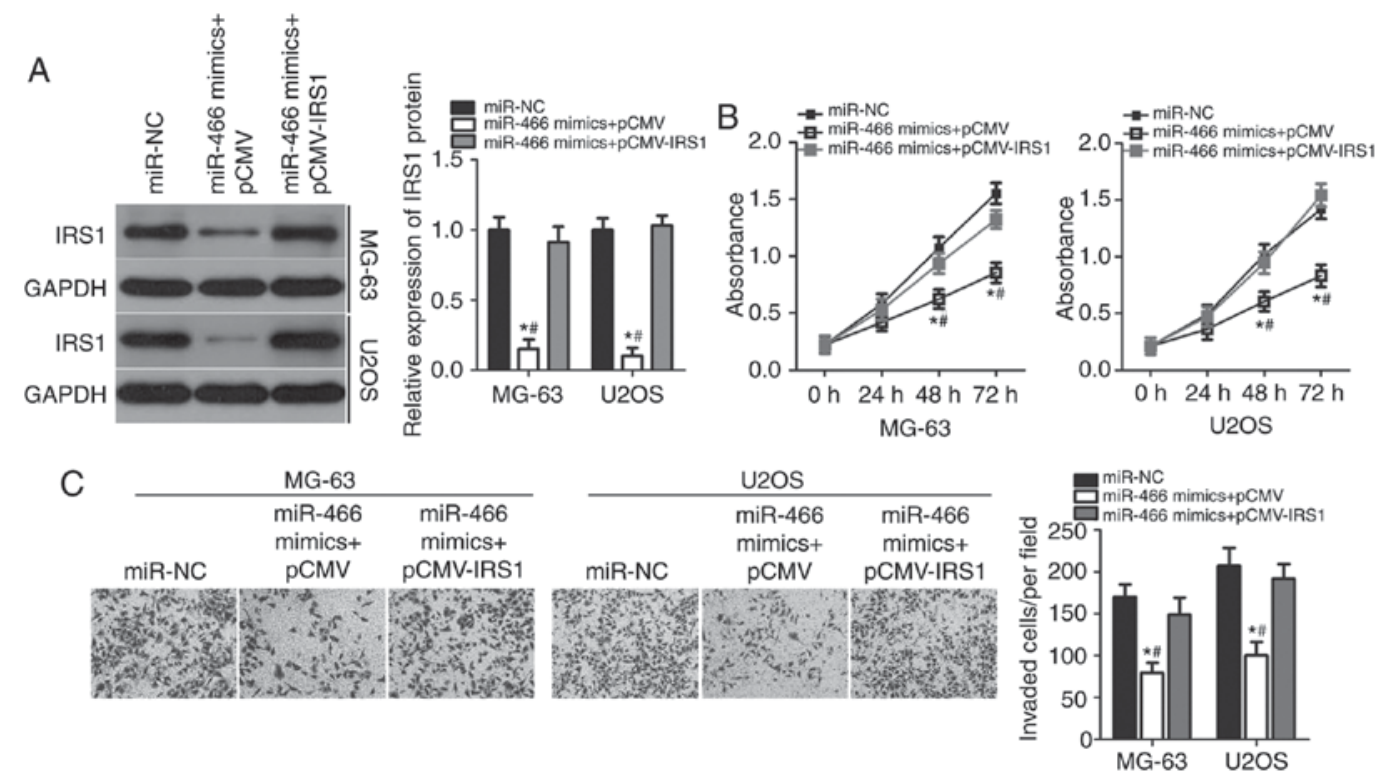

Figure 5. IRS1 overexpression reverses the inhibitory effects of miR-466 overexpression on proliferation and invasion of MG-63 and U2OS cells. (A) MG-63 and U2OS cells overexpressing miR-466 were transfected with empty pCMV plasmid or IRS1 overexpression plasmid without its endogenous 3'-untranslated region. IRS1 protein expression level was determined by western blot analysis. (B) Proliferation and (C) invasion of MG-63 cells were measured by MTT and Matrigel assay, respectively. Magnification, $x 200$. " $\mathrm{P}<0.05$ vs. miR-NC. "P<0.05 vs. miR-466 mimics + pCMV-IRS1. IRS1, insulin receptor substrate 1; miR-, microRNA; NC, negative control; pCMV-IRS1, IRS1 overexpression plasmid.

assays were performed in MG-63 and U2OS cells following co-transfection with miR-466 mimics and empty pCMV or pCMV-IRS1. Overexpression of IRS1 suppressed the inhibitory effects of miR-466 overexpression on the proliferation at 48 and 72 h (Fig. 5B) and invasion (Fig. 5C) of MG-63 and U2OS cells. The present data suggested that miR-466 overexpression inhibited proliferation and invasion of OS cells by decreasing the expression level of IRS1, and that the inhibition of IRS1 was important for the suppressive effects of miR-466 in OS cells.

\section{Discussion}

Accumulating evidence demonstrated that miRNAs are frequently dysregulated in OS, and the aberrant expression of miRNAs is associated with OS initiation and progression (21-23). Therefore, to investigate the expression levels and the functions of miRNAs associated with OS may provide novel insights into OS pathogenesis and may facilitate the identification of novel targets for the diagnosis and treatment of patients with this malignancy. In the present study, the expression level of miR-466 was identified to be downregulated in OS tissues and cell lines. Ectopic overexpression of miR-466 was able to inhibit cell proliferation and invasion in OS cells. Additionally, IRS1 was identified as a direct target of miR-466 in OS cells, and its expression level was significantly upregulated in OS tissues. Notably, the expression levels of IRS1 and miR-466 were inversely correlated in clinical samples of OS. Furthermore, IRS1 overexpression significantly suppressed the inhibitory effects of miR-466 overexpression on the proliferation and invasion of OS cells. The present results suggested that miR-466 may be a novel candidate to develop gene therapy strategies to treat patients with OS.

miR-466 expression has been studied in several types of human malignancies. The expression of miR-466 is downregulated in colorectal cancer tissues and cell lines. Decreased miR-466 expression was identified to be associated with tumor size, Tumor-Node-Metastasis stage, lymph node metastasis and distant metastasis of colorectal cancer patients (16). Patients with colorectal cancer exhibiting a decreased expression level of miR-466 presented decreased overall survival compared with patients with an increased expression level of miR-466. The decreased expression level of miR-466 was identified to be a biomarker associated with poor prognosis in patients with colorectal cancer (16). The expression level of miR-466 was detected to be decreased in prostate cancer tissues and cell lines (17). Receiver operating characteristic curve and Kaplan-Meier analyses demonstrated that the dysregulated expression of miR-466 may represent a biomarker for the diagnosis of prostate cancer (17). In addition, alterations in the expression level of miR-466 may be associated with biochemical relapse in patients with prostate cancer (17). In contrast, the expression level of miR-466 was identified to be upregulated in cervical cancer (18). Increased expression levels of miR-466 were demonstrated to be associated with lymph node metastasis (18). These previous conflicting results suggest that the function of miR-466 may be tissue-specific. Additionally, the expression level of miR-466 may be used as an indicator for the early prognosis of patients with these specific cancer types.

The dysregulation of miR-466 is involved in important processes underlying tumorigenesis and tumor development $(16,17)$. A previous paper identified that miR-466 overexpression inhibited the proliferation and metastasis of colorectal cancer cells (16). Additionally, overexpression of miR-466 induced G0/G1 cycle arrest and promoted apoptosis in colorectal cancer (16). In prostate cancer, miR-466 overexpression inhibited cell proliferation, migration and invasion, causing cell cycle arrest and 
promoting cell apoptosis in vitro. Furthermore, overexpression of miR-466 was able to decrease tumor growth and bone metastasis of prostate cancer in vivo (17). These previous findings suggested that miR-466 may be used as a therapeutic target to effectively inhibit the development of colorectal and prostate cancers.

The investigation of the direct targets of miR-466 in OS may be important in the identification of novel genes involved in OS progression and may further facilitate the development of targeted therapeutic strategies. IRS1 is a mediator of oncogenic IGF signaling, and in the present study, IRS1 was identified to be a direct target of miR-466 in OS. The expression level of IRS1 is increased in multiple malignant tumor types, including breast cancer (24), hepatocellular carcinoma (25), colorectal cancer (26), gastric cancer (27) and non-small cell lung cancer (28). IRS1 serves pro-carcinogenic roles in tumorigenesis and tumor development, and regulates a number of biological features, including cell proliferation, cycle status, apoptosis, metastasis and epithelial-to-mesenchymal transition (28-30). In the present study, the expression level of IRS1 was identified to be upregulated in OS tissues and cell lines. Additionally, inhibition of IRS1 suppressed the proliferation, colony formation ability, migration and invasion of OS cells (20). Due to the roles identified for IRS1 in OS pathogenesis, miR-466-mediated IRS1 gene silencing may represent an effective therapeutic strategy to treat patients with OS.

Collectively, to the best of the authors' knowledge, the present study has identified the tumor-suppressive activity of miR-466 OS. Additionally, the expression level of miR-466 was significantly downregulated in tumor tissues and cell lines, and its overexpression was able to inhibit cell proliferation and invasion. The tumor-suppressive roles of miR-466 in OS cells were mediated by the silencing of IRS1. Therefore, miR-466 may represent a novel potential therapeutic target to treat OS. However, one limitation of the present study was that the sample size was relatively small. Further experiments analyzing data and samples from a larger number of subjects are required to confirm the association between the expression level of miR-466 and the clinicopathological characteristics of patients with OS.

\section{Acknowledgements}

Not applicable.

\section{Funding}

Funding information is not applicable.

\section{Availability of data and materials}

The datasets used and/or analyzed during the present study are available from the corresponding author on reasonable request.

\section{Authors' contributions}

JC designed the present study, and analyzed and interpreted the data. YS and JZ performed the CCK-8 and cell invasion assays. LS and JL conducted RT-qPCR, western blot analysis and luciferase reporter assay. All the authors read and approved the final draft.

\section{Ethics approval and consent to participate}

The present study was approved by The Ethics Committee of The Ningbo No. 6 Hospital, and was performed in accordance with the Declaration of Helsinki and the guidelines of The Ethics Committee of The Ningbo No. 6 Hospital. Written informed consent was obtained from all patients for the use of their clinical tissues.

\section{Patient consent for publication}

Not applicable.

\section{Competing interests}

The authors declare that they have no competing interests.

\section{References}

1. Ottaviani G and Jaffe N: The epidemiology of osteosarcoma. Cancer Treat Res 152: 3-13, 2009.

2. Geller DS and Gorlick R: Osteosarcoma: A review of diagnosis, management, and treatment strategies. Clin Adv Hematol Oncol 8: 705-718, 2010.

3. Ta HT, Dass CR, Choong PF and Dunstan DE: Osteosarcoma treatment: State of the art. Cancer Metastasis Rev 28: 247-263, 2009.

4. Ferrari S and Palmerini E: Adjuvant and neoadjuvant combination chemotherapy for osteogenic sarcoma. Curr Opin Oncol 19: 341-346, 2007.

5. Nouri H, Ben Maitigue M, Abid L, Nouri N, Abdelkader A, Bouaziz M and Mestiri M: Surface osteosarcoma: Clinical features and therapeutic implications. J Bone Oncol 4: 115-123, 2015.

6. Tan ML, Choong PF and Dass CR: Osteosarcoma: Conventional treatment vs. gene therapy. Cancer Biol Ther 8: 106-117, 2009.

7. Hammond SM: An overview of microRNAs. Adv Drug Deliv Rev 87: 3-14, 2015

8. Bartel DP: MicroRNAs: Genomics, biogenesis, mechanism, and function. Cell 116: 281-297, 2004.

9. Yates LA, Norbury CJ and Gilbert RJ: The long and short of microRNA. Cell 153: 516-519, 2013.

10. Tahara H, Kay MA, Yasui W and Tahara E: MicroRNAs in cancer: The 22nd hiroshima cancer seminar/the 4th Japanese Association for RNA interference joint international symposium, 30 August 2012, Grand Prince Hotel Hiroshima. Jpn J Clin Oncol 43: 579-582, 2013.

11. Winter J, Jung S, Keller S, Gregory RI and Diederichs S: Many roads to maturity: microRNA biogenesis pathways and their regulation. Nat Cell Biol 11: 228-234, 2009.

12. Wang X, Lin Y, Peng L, Sun R, Gong X, Du J and Zhang X: MicroRNA-103 promotes proliferation and inhibits apoptosis in spinal osteosarcoma cells by targeting p57. Oncol Res 26: 933-940, 2018.

13. Xiao Y, Zhao Q, Du B, Chen HY and Zhou DZ: MicroRNA-187 inhibits growth and metastasis of osteosarcoma by downregulating S100A4. Cancer Invest 36: 1-9, 2018.

14. Xu Y, Chu H, Zhou Y, Wang J, Dong $\mathrm{C}$ and Yin R: miR-365 functions as a tumor suppressor by directly targeting CYR61 in osteosarcoma. Biomed Pharmacother 98: 531-537, 2018.

15. Calin GA and Croce CM: MicroRNA signatures in human cancers. Nat Rev Cancer 6: 857-866, 2006.

16. Tong F, Ying Y, Pan H, Zhao W, Li H and Zhan X: MicroRNA-466 (miR-466) functions as a tumor suppressor and prognostic factor in colorectal cancer (CRC). Bosn J Basic Med Sci 18: 252-259, 2018.

17. Colden M, Dar AA, Saini S, Dahiya PV, Shahryari V, Yamamura S, Tanaka Y, Stein G, Dahiya R and Majid S: MicroRNA-466 inhibits tumor growth and bone metastasis in prostate cancer by direct regulation of osteogenic transcription factor RUNX2. Cell Death Dis 8: e2572, 2017. 
18. Zhou LL, Shen Y, Gong JM, Sun P and Sheng JH: MicroRNA-466 with tumor markers for cervical cancer screening. Oncotarget 8 : 70821-70827, 2017.

19. Livak KJ and Schmittgen TD: Analysis of relative gene expression data using real-time quantitative PCR and the 2(-Delta Delta C(T)) method. Methods 25: 402-408, 2001.

20. Zhi X, Wu K, Yu D, Wang Y, Yu Y, Yan P and Lv G: MicroR NA-494 inhibits proliferation and metastasis of osteosarcoma through repressing insulin receptor substrate-1. Am J Transl Res 8: 3439-3447, 2016.

21. Ell B and Kang Y: MicroRNAs as regulators of bone homeostasis and bone metastasis. Bonekey Rep 3: 549, 2014.

22. Kafchinski LA and Jones KB: MicroRNAs in osteosarcomagenesis. Adv Exp Med Biol 804: 119-127, 2014.

23. Kim YH, Goh TS, Lee CS, Oh SO, Kim JI, Jeung SH and Pak K: Prognostic value of microRNAs in osteosarcoma: A meta-analysis. Oncotarget 8: 8726-8737, 2017.

24. Porter HA, Perry A, Kingsley C, Tran NL and Keegan AD: IRS 1 is highly expressed in localized breast tumors and regulates the sensitivity of breast cancer cells to chemotherapy, while IRS2 is highly expressed in invasive breast tumors. Cancer Lett 338: 239-248, 2013

25. Lai YY, Shen F, Cai WS, Chen JW, Feng JH, Cao J, Xiao HQ Zhu GH and Xu B: MiR-384 regulated IRS1 expression and suppressed cell proliferation of human hepatocellular carcinoma. Tumour Biol 37: 14165-14171, 2016.
26. Esposito DL, Aru F, Lattanzio R, Morgano A, Abbondanza M, Malekzadeh R, Bishehsari F, Valanzano R, Russo A, Piantelli M, et al: The insulin receptor substrate 1 (IRS1) in intestinal epithelial differentiation and in colorectal cancer. PLoS One 7: e36190, 2012.

27. Zheng H, Zhang F, Lin X, Huang C, Zhang Y, Li Y, Lin J, Chen W and Lin X: MicroRNA-1225-5p inhibits proliferation and metastasis of gastric carcinoma through repressing insulin receptor substrate- 1 and activation of $\beta$-catenin signaling. Oncotarget 7: 4647-4663, 2016.

28. Cao M, Li Y, Lu H, Meng Q, Wang L, Cai L and Dong X: MiR-23a-mediated migration/invasion is rescued by its target, IRS-1, in non-small cell lung cancer cells. J Cancer Res Clin Oncol 140: 1661-1670, 2014

29. Wang Y, Zhang X, Zou C, Kung HF, Lin MC, Dress A, Wardle F, Jiang BH and Lai L: miR-195 inhibits tumor growth and angiogenesis through modulating IRS1 in breast cancer. Biomed Pharmacother 80: 95-101, 2016.

30. Wang Y, Hu C, Cheng J, Chen B, Ke Q, Lv Z, Wu J and Zhou Y: MicroRNA-145 suppresses hepatocellular carcinoma by targeting IRS1 and its downstream Akt signaling. Biochem Biophys Res Commun 446: 1255-1260, 2014. 